\title{
Fabrication and Sub-Band-Gap Absorption of Single-Crystal Si Supersaturated with Se by Pulsed Laser Mixing
}

\section{Citation}

Tabbal, Malek, Taegon Kim, David N. Woolf, Byungha Shin, and Michael J. Aziz. 2010. Fabrication and sub-band-gap absorption of single-crystal Si supersaturated with Se by pulsed laser mixing. Applied Physics A: Materials Science \& Processing 98(3): 589-594.

\section{Published Version}

doi:10.1007/s00339-009-5462-1

\section{Permanent link}

http://nrs.harvard.edu/urn-3:HUL.InstRepos:4341783

\section{Terms of Use}

This article was downloaded from Harvard University's DASH repository, and is made available under the terms and conditions applicable to Other Posted Material, as set forth at http:// nrs.harvard.edu/urn-3:HUL.InstRepos:dash.current.terms-of-use\#LAA

\section{Share Your Story}

The Harvard community has made this article openly available.

Please share how this access benefits you. Submit a story.

\section{Accessibility}




\title{
Fabrication and sub-band-gap absorption of single-crystal $\mathrm{Si}$ supersaturated with Se by pulsed laser mixing
}

\author{
Malek Tabbal • Taegon Kim • David N. Woolf • \\ Byungha Shin • Michael J. Aziz
}

Received: 25 March 2009 / Accepted: 20 October 2009 / Published online: 12 November 2009

(c) Springer-Verlag 2009

\begin{abstract}
Selenium supersaturated silicon layers were fabricated by pulsed excimer laser induced liquid-phase mixing of thin Se films on Si(001) wafers. Sufficiently low Se coverage avoids destabilization of rapid epitaxial solidification, resulting in supersaturated solid solutions free of extended defects, as shown by transmission electron microscopy. The amount of retained Se depends on the original film thickness, the laser fluence, and the number of laser pulses irradiating the same spot on the surface. Using this method, Se has incorporated into the topmost $300 \mathrm{~nm}$ of the silicon with a concentration of 0.1 at.\%. Channeling Rutherford backscattering spectrometry measurements show that the substitutional fraction can be as high as $75 \%$ of the total retained Se. These alloys exhibit strong sub-band-gap absorption with optical absorption coefficient ranging up to about $10^{4} \mathrm{~cm}^{-1}$, thus making them potential candidates for applications in Si-based optoelectronic devices.
\end{abstract}

PACS $87.15 . \mathrm{Nt} \cdot$ 78.66.Db $\cdot$ 79.20.Ds

\section{Introduction}

Silicon heavily doped with chalcogens represent a new class of materials that show significant below band-gap infrared absorption [1], making them interesting for sub-band-gap

\footnotetext{
M. Tabbal (凶)

Department of Physics, American University of Beirut, Beirut 1107 2020, Lebanon

e-mail: malek.tabbal@aub.edu.lb

M. Tabbal · T. Kim · D.N. Woolf · B. Shin · M.J. Aziz Harvard School of Engineering and Applied Sciences, Cambridge, MA 02138, USA
}

optoelectronic applications [2-4]. This material can be prepared [5] by irradiation of Si by femtosecond or nanosecond laser pulses in a chalcogen containing atmosphere such as $\mathrm{SF}_{6}$ leading to a spiked chalcogen-bearing Si surface with a near-unity sub-band-gap absorptance. More recently, alternative fabrication methods have been developed, such as spreading a powder of the chalcogen dopants $\mathrm{S}, \mathrm{Se}$, and Te [6] or evaporation of chalcogen thin films [7] on the Si surface and subsequent femtosecond laser irradiation. It was found that the sub-band-gap absorptance is greater and more resistant to annealing for Se and Te than for S-doped Silicon. A flat (nonspiked) form of chalcogen supersaturated Si was also synthesized by ion implantation followed by pulsed laser melting by a few spatially uniform excimer laser shots $[8,9]$. This flat form of supersaturated $\mathrm{Si}$ also demonstrated considerable sub-band-gap absorption and could be more adaptable than its spiked counterpart for integration in device fabrication.

One technique that could provide a low cost and more flexible alternative to the ones based on ion implantation is the incorporation of dopants or alloying elements through the melting and mixing of a thin vapor-deposited film with the underlying $\mathrm{Si}$ substrate induced by a few high-power laser pulses. Under suitably chosen conditions of laser irradiation, the subsequent rapid solidification could leave a supersaturated high-quality single crystal material that often cannot be obtained using more conventional heating techniques, such as furnace or rapid thermal annealing. This so-called "pulsed laser mixing" (PLM) technique has been successfully utilized to form heteroepitaxial SiGe alloys [10, 11]. It has also been used to incorporate high level of substitutional $\mathrm{Sb}$ into $\mathrm{Si}$ but at the cost of a defective, cellular solidification morphology [12]. In this paper, we report on the fabrication of Selenium (Se) supersaturated silicon ( $\mathrm{Si}$ ) layers by excimer laser induced mixing of 
Se layers controllably evaporated on Si wafers and limited to such small thicknesses that single-phase, supersaturated single-crystal solidification without cellular morphological breakdown [13] is enabled. The Se content, concentration depth profile and substitutional fraction were determined by Rutherford backscattering spectrometry (RBS), and their crystalline quality was evaluated using cross-section transmission electron microscopy. The optical absorptance and reflectance were investigated over the range 900-2500 nm and the optical absorption coefficient in the sub-band-gap region was determined.

\section{Experimental details}

Pure Se shot (Puratronic, purity 99.999\%) was used as a source material to deposit thin Se layers on single-crystal p-type-Si(001) (B-doped, $5 \Omega \mathrm{cm}, \sim 10^{15} \mathrm{~cm}^{-3}$ ) substrates, by thermal evaporation in vacuum, with a base pressure below $10^{-6}$ Torr. Prior to deposition, the substrates were conventionally cleaned and the surface native oxide was removed by dipping in a 1:10 $\mathrm{HF}: \mathrm{H}_{2} \mathrm{O}$ solution. Evaporation currents were kept very low, and the Se growth rate, estimated to be about $1 \mathrm{~nm} / \mathrm{min}$, was monitored by means of a quartz-crystal thickness monitor. Films with thicknesses (as deduced from RBS measurements) of 1, 2.2, and $4.5 \mathrm{~nm}$ were thus obtained. The samples were then irradiated by one or ten laser shots, in ambient air, using a spatially homogenized pulsed $\mathrm{XeCl}^{+}$excimer laser beam (308 nm, $25 \mathrm{~ns}$ FWHM, 50-ns total duration) with fluences ranging between 0.6 and $1.4 \mathrm{~J} / \mathrm{cm}^{2}$, well below the surface damage threshold of the single $\mathrm{Si}$ wafers that is estimated to be above $2.3 \mathrm{~J} / \mathrm{cm}^{2}$. All laser irradiated films appeared smooth and defect-free to the naked eye and no ablation plume was seen during irradiation. The excimer laser spot size ranged between $3 \times 3$ to $5 \times 5 \mathrm{~mm}^{2}$ depending on focusing conditions. Time-resolved reflectivity (TRR) of a low-power $\mathrm{Ar}^{+}$ ion laser $(488 \mathrm{~nm})$ confirmed an optically flat surface and allowed us to monitor the duration over which the surface was molten. The $\mathrm{Ar}^{+}$ion laser beam is incident near grazing angle on the Si surface and its reflected beam is detected by a fast photodiode that is connected to a digital oscilloscope in order to record the temporal variation of the reflected intensity. TRR was also used to calibrate the laser fluence by measuring the melt duration for a virgin crystalline $\mathrm{Si}$ wafer and comparing to predictions of the melt depth versus fluence by a numerical solution of the one-dimensional heat equation using the well-established optical and thermophysical properties of silicon [14].

The RBS measurements were performed using $2-\mathrm{MeV}$ $\mathrm{He}^{+}$beam incident (with spot diameter less than $1 \mathrm{~mm}$ ) perpendicular to the sample surface, and the $180^{\circ}$ backscattered yield was collected by an annular detector with an energy resolution close to $20 \mathrm{keV}$. For some selected samples, the Se depth profiles and substitutional fraction of incorporated Se were quantified by performing channeling mode RBS (c-RBS) with the $2-\mathrm{MeV} \mathrm{He}^{+}$beam aligned with the [110] axis at an angle of $45^{\circ}$ from the sample's [001] surface normal, in conjunction with nonchanneling RBS measurements with the scattering geometry unchanged but the sample rotated about its surface normal. For these measurements, a passivated implanted planarized silicon (PIPS) detector, having an energy resolution of $13 \mathrm{keV}$, was placed in a direction coplanar with the incoming beam and the surface normal, and at an angle of $22.5^{\circ}$ from grazing the sample's surface in order to detect ions backscattered through $157.5^{\circ}$. The thickness of the as-evaporated films and the total Se concentration in the Si substrate after laser irradiation were determined by simulation of the RBS spectra using the software packages RUMP [15] and SA [16] to determine the depth profile of the Se concentration and its substitutional fraction. The crystalline quality of the laser melted layers was evaluated by cross-sectional transmission electron microscopy (XTEM) carried out at $200 \mathrm{kV}$ using a JEOL 2010F electron microscope, with lattice imaging obtained along the Si [110] direction. Total hemispherical specular and diffuse reflectance, $R$, and transmittance, $T$, over the range 900-2500 nm were measured using a Hitachi U-4001 UV-VIS spectrophotometer equipped with an integrating sphere detector. A series of lenses and diaphragms was used to reduce the spot size of the incident light beam to an area less than $2 \times 2 \mathrm{~mm}^{2}$, and the beam was carefully aligned to the center of the sample's laser irradiated area. The validity of this small area measurement was confirmed by comparison with a large area measurement for a sample with well-known reflectance and transmission curves.

\section{Results}

Figure 1 shows the RBS Se peak of a 2.2-nm thick layer evaporated on $\mathrm{Si}$ before laser irradiation and after irradiation at $0.6 \mathrm{~J} / \mathrm{cm}^{2}$ with a single laser shot (a complete spectrum for which is also shown in the inset) and ten shots. This particular fluence was chosen as it is just above the melting threshold of crystalline silicon determined from the laser reflectometry measurements, and is the lowest fluence used in this work. The decrease in the area under the Se RBS peak indicates the amount of Se loss caused by laser irradiation. This effect could be attributed to ablation caused by the high-power laser pulse or vaporization induced by the rapid temperature increase at the surface of the sample. Qualitatively, this effect was observed at all laser fluences $(0.6,1.0$, and $1.4 \mathrm{~J} / \mathrm{cm}^{2}$ ) and for all three initial thicknesses, namely 1, 2.2, and $4.5 \mathrm{~nm}$, as reported in Fig. 2. Quantitatively, the 


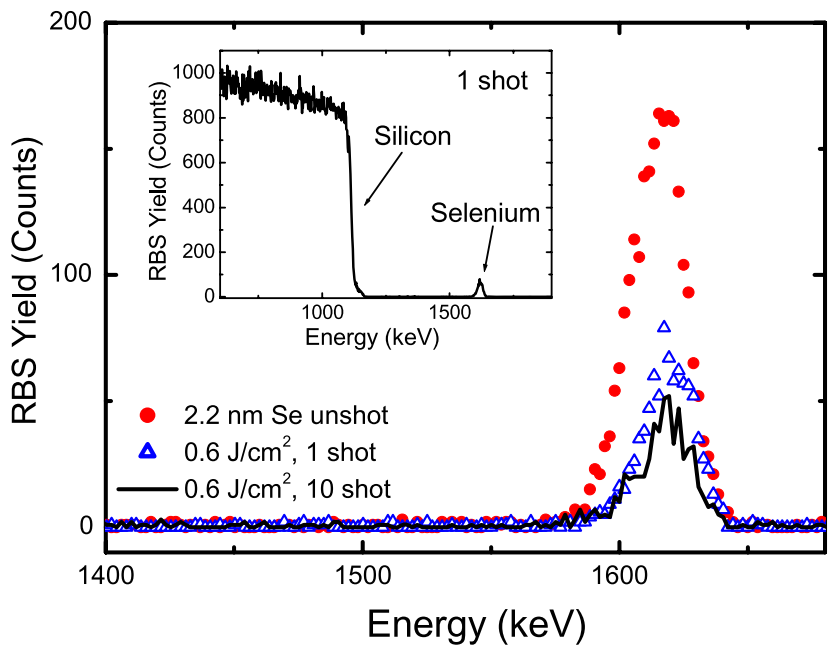

Fig. 1 RBS Se peaks of a 2.2-nm thick layer evaporated on $\mathrm{Si}$, (a) before laser irradiation (red dots), and after irradiation at $0.6 \mathrm{~J} / \mathrm{cm}^{2}$ with (b) a single laser shot (open triangles) and (c) ten shots (solid line). A complete RBS spectrum of sample irradiated at $0.6 \mathrm{~J} / \mathrm{cm}^{2}$ with a single laser shot is also shown in the inset of the figure

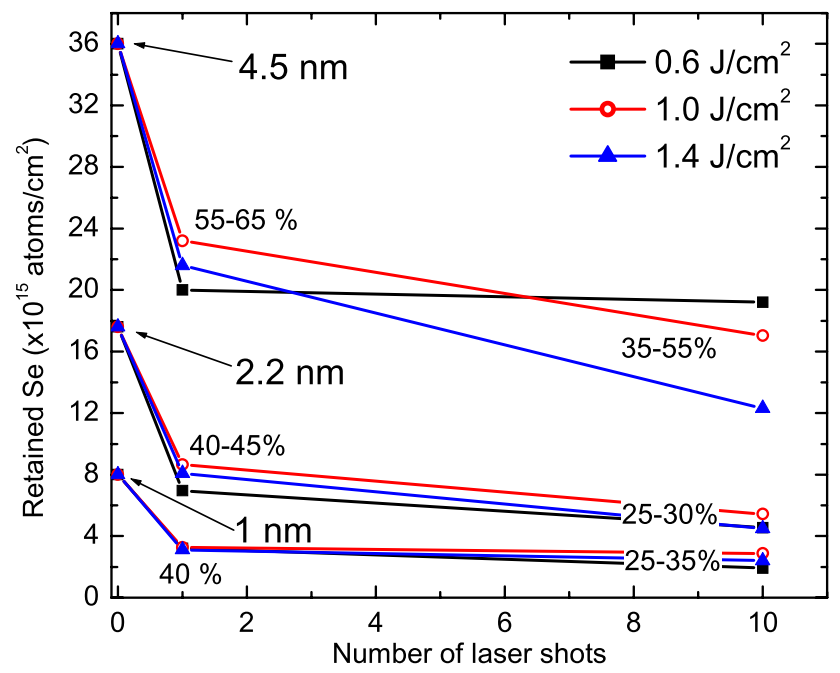

Fig. 2 Effect of number of laser shots on the Se concentration in the layers, determined by RBS, for three different laser fluences $(0.6,1$, and $1.4 \mathrm{~J} / \mathrm{cm}^{2}$ ) and three different initial thicknesses of the evaporated $\mathrm{Se}(1,2.2$, and $4.5 \mathrm{~nm})$. The percentages reported on the figure represent the amount of Se retained after laser irradiation

results vary from sample to sample depending on the initial evaporated thickness and, to a lesser extent, on laser fluence. Indeed, for the thinner layers ( 1 and $2.2 \mathrm{~nm}$ ), irradiation with a single laser shot leads to retention of $40-45 \%$ of the initially deposited Se whereas $55-65 \%$ is retained for the thicker film $(4.5 \mathrm{~nm})$. After irradiation with ten laser shots, the amount of retained Se ranges from $25-35 \%$ for the thinner ( 1 and $2.2 \mathrm{~nm}$ ) layers up to $35-55 \%$ for the thicker $(4.5 \mathrm{~nm})$ layers. Therefore, the amount of Se lost per laser shot significantly drops after multiple irradiation, presumably as a result of a deeper diffusion of Se atoms into the

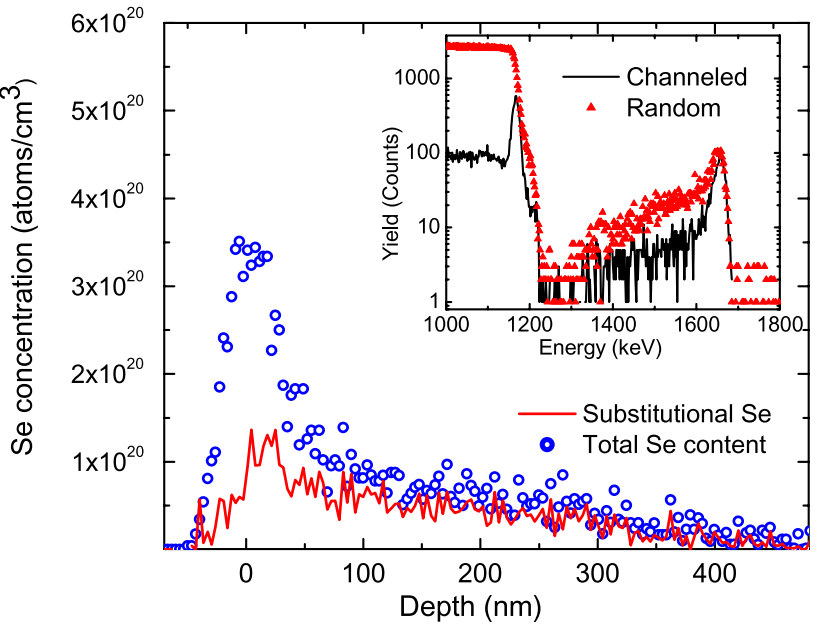

Fig. 3 Depth profile of the total and substitutional Se in Si for the sample coated with $2.2 \mathrm{~nm}$ and irradiated with ten laser shots at a fluence of $1.4 \mathrm{~J} / \mathrm{cm}^{2}$. The profiles were determined from the random and channeling RBS spectra shown in the inset

Si substrate. This is supported by the broadening of the Se concentration-depth profiles with multiple laser shots, particularly for films irradiated at a fluence of $1.4 \mathrm{~J} / \mathrm{cm}^{2}$ as is going to be discussed. However, there is no clear trend relating Se loss to laser fluence, possibly because of the presence of two competing processes both of which would be enhanced at higher laser fluence: Se vaporization/ablation versus Se diffusion into the Si substrate while molten. Only the thickest film irradiated with ten laser shots shows steadily increasing Se loss with laser fluence. It is possible that the near-surface concentration from the thickest deposited film is high enough to favor vaporization/ablation even after ten laser shots.

In Fig. 3 we show the depth profile deduced from grazing exit RBS random and [110] channeled spectra for the sample coated with $2.2 \mathrm{~nm}$ of Se and irradiated with ten shots at a laser fluence of $1.4 \mathrm{~J} / \mathrm{cm}^{2}$. In addition to the surface peak, a significant Se incorporation, evaluated at about $5 \times 10^{19}$ atoms $/ \mathrm{cm}^{3}$, is detected down to a depth exceeding $300 \mathrm{~nm}$. For the sample initially coated with $1 \mathrm{~nm}$ (not shown) and irradiated with ten shots at a laser fluence of $1.4 \mathrm{~J} / \mathrm{cm}^{2}$, a Se content of about $3 \times 10^{19}$ atoms $/ \mathrm{cm}^{3}$ was measured down to similar depths. In both cases, the measured Se concentration is greater by at least two orders of magnitude the equilibrium solubility limit, which does not exceed $10^{17}$ atoms $/ \mathrm{cm}^{3}$ [17]. The supersaturation arises by Se diffusion and mixing during the period the $\mathrm{Si}$ is molten, and a substantial degree of solute trapping by the rapidly moving solidification front [14]. Channeling RBS spectra, taken under grazing incidence geometry, were used to estimate the substitutional fraction of Se as a function of depth, $f(z)$ in the usual manner, as also presented in Fig. 3, with

$f(z)=1-\chi_{\min }^{\mathrm{Se}}(z) / \chi_{\min }^{\mathrm{Si}}(z)$, 


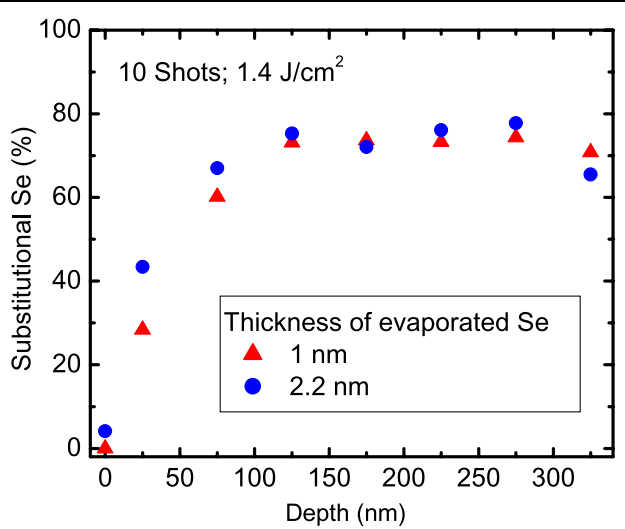

Fig. 4 Variation of the substitutional percentage of Se as a function of depth in the Si susbtrate, for two different initial thicknesses of evaporated Se. Both samples were irradiated with ten laser shots at a fluence of $1.4 \mathrm{~J} / \mathrm{cm}^{2}$

where $\chi_{\min }^{\mathrm{Se}}$ and $\chi_{\min }^{\mathrm{Si}}$ represent, for each element, the ratio of counts in the channeling spectrum to that in the random spectrum at depth $z$. The number of counts in the channeled and random spectra is determined from the raw data shown in the inset of Fig. 3, as a function of energy, and then converted to depth by depth-calibration of the energy scale [16]. In that manner, the substitutional fraction, averaged over 50-nm intervals, was evaluated as a function of depth, for the samples initially coated with 1- and 2.2-nm thick Se layers and laser melted with ten shots at a fluence of $1.4 \mathrm{~J} / \mathrm{cm}^{2}$, as shown in Fig. 4. For both samples, the substitutional fraction of Se is quite low near the surface but it increases rapidly as a function of depth and reaches a nearly constant value of $75 \%$ at a depth of $100 \mathrm{~nm}$ and beyond. However, at a depth greater than $350 \mathrm{~nm}$, the Se signal becomes too weak to deduce a meaningful value of the substitutional fraction. The depth to which the Se penetrates into the silicon is limited by the maximum melt depth, a well-known effect for pulsed laser melting of supersaturated Si [14]. For multiply-shot samples there is likely to be sufficient cumulative melt duration for Se to diffuse throughout the melted region. Hence we tentatively identify the penetration depth of 300-400 nm as the maximum melt depth. With ion-implanted silicon, well-calibrated numerical solutions of the 1-D heat equation ("simulations") permit an estimate of the melt depth [14]. Uncertainties in the absorbed fluence cause the greatest uncertainty in the predicted melt depth but this uncertainty is removed by adjusting the fluence to match the melt duration determined by time-resolved reflectometry. We were unable to follow this procedure for samples with evaporated Se surface films - even very thin ones-because the interpretation of the surface reflectometry was ambiguous, the optical and thermophysical properties of the Se film are not well known, and we do not account for the Se lost to evaporation/ablation in the simulation.

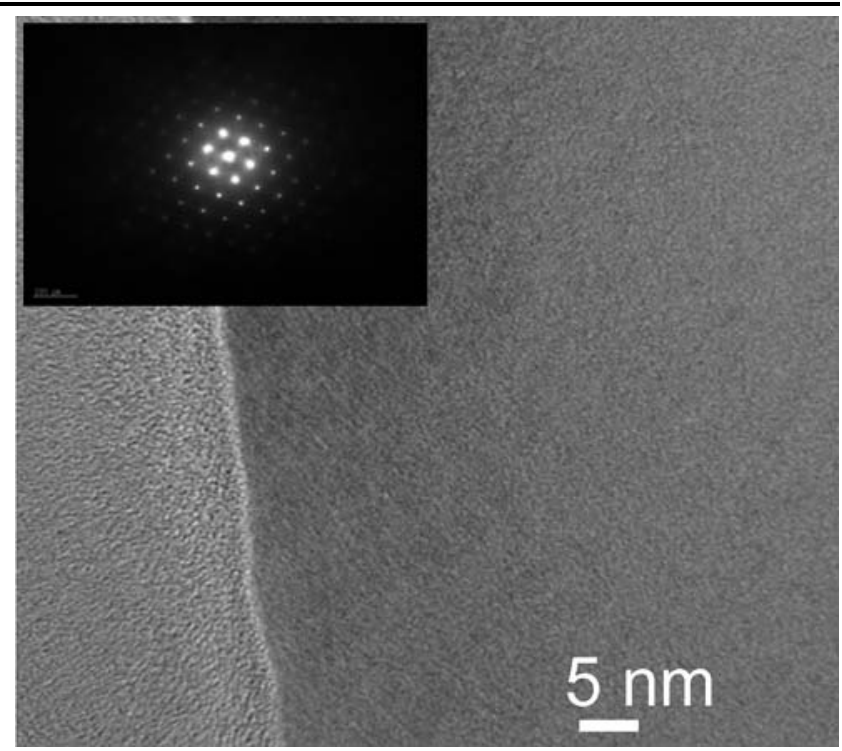

Fig. 5 High-resolution cross-sectional TEM image of the sample initially coated with 2.2-nm thick Se film and irradiated with ten laser shots at a fluence of $1.4 \mathrm{~J} / \mathrm{cm}^{2}$. The sample appears dark, while the lighter region occupying the leftmost quarter of the image is the glue used to hold the sample. A selected-area diffraction pattern taken from the resolidified region of the sample is shown in the inset

The high crystalline quality of the pulsed laser melted layers is confirmed by the high-resolution XTEM image shown in Fig. 5, for the sample with an initially 2.2-nm thick Se layer and irradiated with ten laser shots at a fluence of $1.4 \mathrm{~J} / \mathrm{cm}^{2}$. A selected area diffraction pattern of the sample, shown in the inset of the figure, consists of sharp diffraction spots solely corresponding to single crystal $\mathrm{Si}$, so that no secondary phases or precipitates are detected. In addition, the real space images, of which Fig. 5 is an example, indicate the absence of any extended defects, such as dislocations or cell walls. The latter result from a morphological instability of the solid liquid interface during solidification at sufficiently high solute concentration and appear by diffraction contrast [13]. In contrast to previous work on thicker dopant films [12], our approach of using very thin evaporated films was apparently successful in avoiding the formation of cell walls.

Figure 6 shows the transmittance and reflectance spectra of the sample obtained by laser mixing of a $2.2-\mathrm{nm} \mathrm{Se}$ film into the Si substrate. The transmittance and reflectance spectra of the bare silicon substrate are also presented, and the fundamental absorption band gap of $\mathrm{Si}$ is evident near a wavelength $\lambda \approx 1200 \mathrm{~nm}$. At sub-band-gap wavelengths $(\lambda>1200 \mathrm{~nm})$, the Si reflectance and transmittance are close to $\sim 0.5$, with no measurable absorption. For the laser mixed sample, both reflectance and transmittance decrease noticeably over a range extending to the highest wavelength accessible to the spectrophotometer, namely $2500 \mathrm{~nm}$. The difference that appears between the transmittance and the reflectance curves of the laser melted samples seen in Fig. 6 


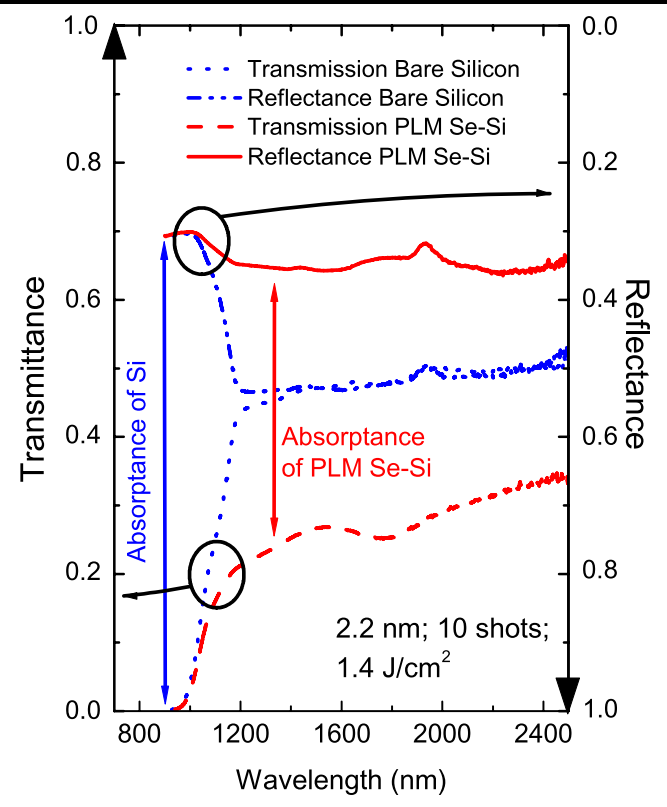

Fig. 6 Optical transmittance and reflectance curves as a function of wavelength of the sample obtained by pulsed laser mixing (PLM, ten laser shots at a fluence of $1.4 \mathrm{~J} / \mathrm{cm}^{2}$ ) of a $2.2-\mathrm{nm}$ thick Se film into the $\mathrm{Si}$ substrate and for the bare silicon substrate. Reflectance scale is inverted so the absorptance can be evaluated graphically

is a measure of the absorptance. Thus, the laser melted sample shows significant broad and nearly flat sub-band-gap absorption. For $\lambda>1200 \mathrm{~nm}$, where absorption from the substrate is negligible, we estimate the absorption coefficient, $\alpha$, of the Se containing laser melted Si layers as

$\alpha=(1 / d) \ln [(1-R) / T]$,

where $d$ is the thickness of the layer containing a significant amount of Se, (estimated to be about $300 \mathrm{~nm}$ from the RBS measurements) while $R$ and $T$ are the directly measured reflectance and transmittance. A value of $3 \times 10^{4} \mathrm{~cm}^{-1}$ for $\alpha$ was thus calculated for a wavelength of $1500 \mathrm{~nm}$. This value is of the same order of magnitude as those reported for laser-melted sulfur-implanted Si [8,9] with comparable dopant levels. It was shown that such very high values of $\alpha$ cannot be explained by free carrier absorption even if we assume complete electrical activation of the dopants. Se, like Sulfur, is a deep donor with levels as low as $0.59 \mathrm{eV}$ below the conduction band edge of Si [18]. Therefore, electrical activation is expected to be much lower than in the case of shallow donors such as arsenic. Indeed, recent measurements on laser melted $\mathrm{Si}$ supersaturated with $\mathrm{S}$ indicate that dopant activation could be lower than $10 \%$ [4]. Thus, there is a different absorption mechanism than free carrier absorption, most likely involving states in the band gap, causing the observed sub-band-gap infrared absorption in our samples.

Figure 7 summarizes the effect of laser fluence and Se content on sub-band-gap absorption. The total absorptance

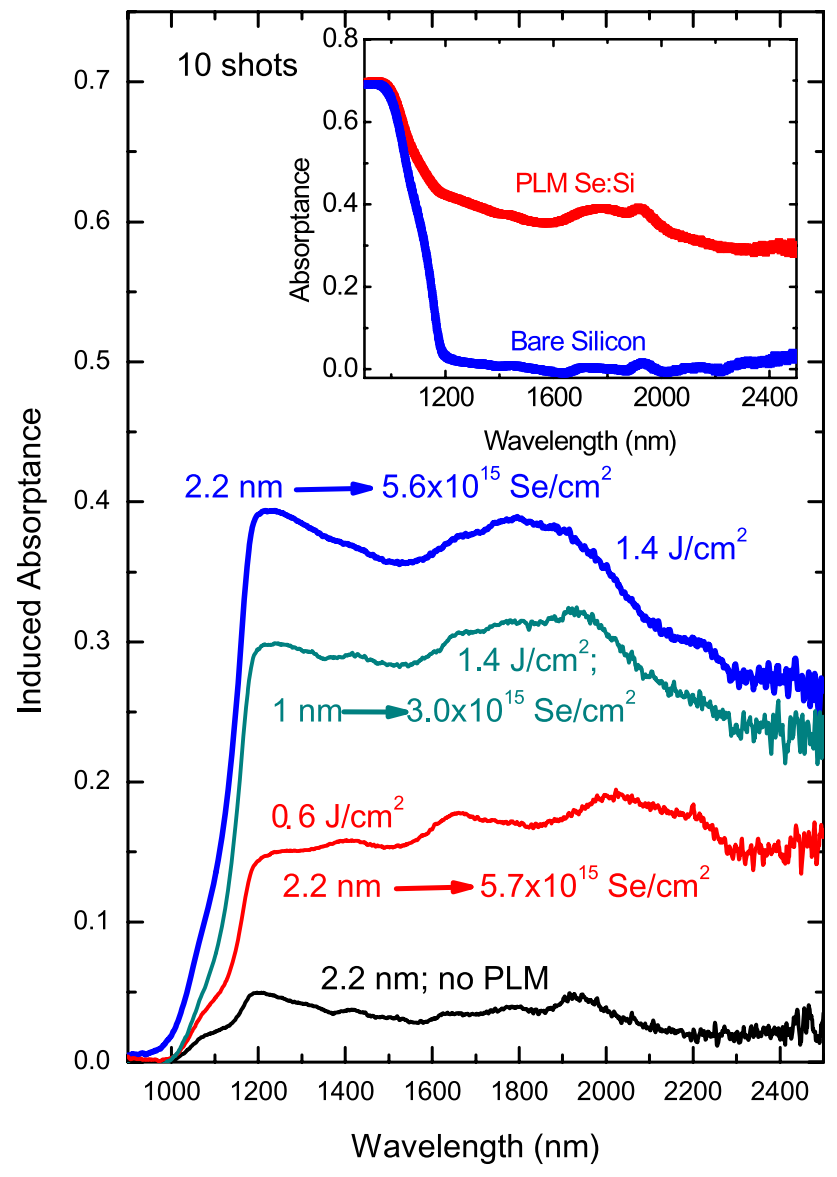

Fig. 7 Induced absorptance data from the pulsed laser mixed samples at different laser fluence and different initial thickness of evaporated Se. The data corresponding to a nonirradiated 2.2-nm Se film on silicon is also shown. Inset: raw absorptance from Fig. 6

$A(A=1-T-R)$ was determined from the directly measured transmittance and reflectance (as shown in Fig. 6), and the curves corresponding to the samples presented in Fig. 6 (i.e., bare silicon and a laser melted sample) are shown in the inset. The difference between the absorptance curves of the laser mixed sample and the bare Silicon substrate is then plotted as the "induced absorptance" in Fig. 7, and is a direct measure of the absorptance of the laser-melted supersaturated layers. The induced absorptance is also shown for the nonirradiated 2.2-nm thick Se film on $\mathrm{Si}$, for the sake of comparison, demonstrating that Se retained in solid solution in Si has much higher absorptance than a greater amount of Se in a deposited surface film. All ten-shot laserirradiated samples exhibit strong, broad sub-band-gap absorption. Comparison of the 2.2-nm film $\left(5.6 \times 10^{15} \mathrm{Se} / \mathrm{cm}^{2}\right.$ retained) and the $1-\mathrm{nm}$ film $\left(3.0 \times 10^{15} \mathrm{Se} / \mathrm{cm}^{2}\right.$ retained $)$ irradiated by $1.4 \mathrm{~J} / \mathrm{cm}^{2}$ shows a sub-band-gap absorptance ratio (over the range 1200-2000 $\mathrm{nm}$ ) scaling approximately as the retained Se content (Fig. 2) and, as deduced from Fig. 4, as the substitutional Se. The 2.2-nm film irradiated with $1.4 \mathrm{~J} / \mathrm{cm}^{2}$ exhibits a substantially higher sub-band- 
gap absorptance than the identical sample irradiated with $0.6 \mathrm{~J} / \mathrm{cm}^{2}$, even though the amount of retained Se is the same in both cases. It is possible that, although they have the same retained Se content, identical films irradiated with different fluences have different substitutional Se content due to the differences in melt duration, melt depth, and solidification rate.

The samples irradiated at $1.4 \mathrm{~J} / \mathrm{cm}^{2}$ seem to exhibit in Fig. 7 a broad peak centered at $\sim 1800 \mathrm{~nm}$, superposed on top of a strong, smooth, sloping baseline of sub-bandgap absorption. While the sub-band-gap absorption phenomenon is still not understood, dilute chalcogen atoms are known to form deep levels in the silicon band gap [18]. It has been proposed that, due to the high concentration of substitutional chalcogen incorporation, impurity bands within the $\mathrm{Si}$ forbidden gap may form and lead to the observed broad sub-band-gap absorption [8, 19].

\section{Summary}

We have demonstrated the synthesis of selenium supersaturated silicon single crystal layers by pulsed laser induced mixing of evaporated thin Se films on $\mathrm{Si}(001)$ wafers. In this process, films of Se with single-digit nanometer thickness were controllably evaporated onto the Si surface and laser melted using 1 to 10 excimer laser pulses at fluences ranging from 0.6 to $1.4 \mathrm{~J} / \mathrm{cm}^{2}$. RBS analysis shows that the process forms supersaturated solutions to a depth of about $300 \mathrm{~nm}$, with a concentration of $\sim 0.1$ at.\%, which is more than two orders of magnitude greater than the equilibrium solubility limit. RBS measurements under channeling geometry show that as much as $75 \%$ of the dissolved Se atoms are incorporated into substitutional sites. The rapid melting and solidification inherent to the process enables solute trapping to form a supersaturated single crystal free from extended defects or any detectable cellular breakdown, as indicated by TEM. Optical characterization shows that this material exhibits strong sub-band-gap infrared absorption, with optical absorption coefficient ranging up to about $10^{4} \mathrm{~cm}^{-1}$, thus making it of interest for applications in Si-based optoelectronic devices. This pulsed laser mixing technique to fabricate supersaturated silicon-based materials could prove to be a viable alternative to the more elaborate techniques based on ion implantation whenever a solid film can be controllably deposited that is sufficiently thin to permit defect-free solidification from PLM.

Acknowledgements We thank J.E. Carey, E. Mazur, B. Tull, J.M. Warrender, and M. Winkler for helpful discussions, and F. Zhao and D. Bell for the TEM measurements. We also thank J. Chervinsky for technical support for the RBS measurements. This research was supported in part by the US Army-ARDEC under contract W15QKN07-P-0092. One of the authors (M.T.) acknowledges financial support from the Fulbright and Arab Fund programs. T.G.K. was supported by the Korea Research Foundation Grant funded by the Korean Government (MOEHRD, Basic Research Promotion Fund), KRF-2004-214C00156. Central facilities were used within the Harvard Center for Nanoscale Systems.

\section{References}

1. C. Wu, C.H. Crouch, L. Zhao, J.E. Carey, R. Younkin, J.A. Levinson, E. Mazur, R.M. Farrell, P. Gothoskar, A. Karger, Appl. Phys. Lett. 78, 1850 (2001)

2. J.E. Carey, C.H. Crouch, M.A. Sheehy, M. Shen, C.M. Friend, E. Mazur, Opt. Lett. 30, 1773 (2005)

3. Z. Huang, J.E. Carey, M. Liu, X. Guo, E. Mazur, J.C. Campbell, Appl. Phys. Lett. 89, 033506 (2006)

4. M. Tabbal, T.G. Kim, J.M. Warrender, M.J. Aziz, B.L. Cardozo, R.S. Goldman, J. Vac. Sci. Technol. B 25, 1747 (2007)

5. C.H. Crouch, J.E. Carey, J.M. Warrender, E. Mazur, M.J. Aziz, F. Genin, Appl. Phys. Lett. 84, 1850 (2004)

6. M.A. Sheehy, B.R. Tull, C.M. Friend, E. Mazur, Mater. Sci. Eng. B-Solid State Mater. Adv. Technol. 137, 289 (2007)

7. B.R. Tull, Ph.D. Thesis, Harvard University (2007)

8. T.G. Kim, J.M. Warrender, M.J. Aziz, Appl. Phys. Lett. 88, $241902(2006)$

9. M. Tabbal, M.J. Aziz, C.S. Madi, S. Charnvanichborikarn, J.S. Williams, T.C. Christidis, Proc. SPIE 6458, 645803 (2007)

10. R. Larciprete, M.G. Grimaldi, E. Borsella, S. Cozzi, S. Martelli, S. Pieretti, I. Vianey, J. Vac. Sci. Technol. A 16, 1589 (1998)

11. K.-J. Kramer, S. Talwar, T.W. Sigmon, K.H. Wiener, Appl. Phys. Lett. 61, 769 (1992)

12. E.P. Fogarassy, D.H. Lowndes, J. Narayan, C.W. White, J. Appl. Phys. 58, 2167 (1985)

13. D.E. Hoglund, M.O. Thompson, M.J. Aziz, Phys. Rev. B 58, 189 (1998)

14. R. Reitano, P.M. Smith, M.J. Aziz, J. Appl. Phys. 76, 1518 (1994)

15. L.R. Doolittle, Nucl. Instrum. Methods B 15, 227 (1986)

16. P.M. Smith, Ph.D. Thesis, Harvard University (1992)

17. A.A. Taskin, B.A. Zaitsev, V.I. Obodnikov, E.G. Tishkovskii, Semiconductors 34, 312 (2000)

18. E. Janzen, R. Stedman, G. Grossmann, H.G. Grimmeiss, Phys. Rev. B 29, 1907 (1984)

19. M.A. Sheehy, L. Winston, J.E. Carey, C.M. Friend, E. Mazur, Chem. Mater. 17, 3582 (2005) 